\title{
Bias factor in anisotropic stochastic fields
}

\author{
M Bahrami Nasr ${ }^{2}$ and S M S Movahed ${ }^{1,2,3}$ \\ 1. Department of Physics, Shahid Beheshti University, Velenjak, Tehran, Iran \\ 2. Ibn Sina lab. Department of Physics, Shahid Beheshti University, Velenjak, Tehran, Iran \\ 3. School of Physics, Institute for Research in Fundamental Sciences (IPM), Tehran, Iran
}

E-mail: m.s.movahed@ipm.ir

(Received 11 June 2019 ; in final form 20 September 2019)

\begin{abstract}
The geometrical and topological measures enable us to characterize stochastic field systematically, and the relation between weighted and unweighted N-point functions is provided. One of such relations is given by the bias factor. In this paper, based on peak statistics, we study the bias factor for stochastic and anisotropic fields. Accordingly, we present the analytical description of local and linear bias factor. Doing simulations, we examine the validation of the derived analytical relation. Our results show that at high threshold level and large spatial separation, there exists a good agreement between the analytical calculation and numerical computations.
\end{abstract}

Keywords: stochastic field, bias factor, anisotropy, geometrical and topological features

For full article, refer to the Persian section 